\title{
Drop impact of a typical portable electronic device
}

\author{
C. Y. Zhou, T. X. Yu \& R. S. W. Lee \\ Department of Mechanical Engineering, \\ Hong Kong University of Science and Technology Clear Water Bay, \\ Kowloon, Hong Kong, China
}

\begin{abstract}
Drop impact reliability is an important concern for the design and use of portable electronic products. When the product is accidentally dropped on the ground, impact forces are transmitted from the product case to the printed circuit board (PCB) and other components within the case. These forces may cause severe functional damage in the form of component failure and/or interconnection breakage. This paper reports our investigation on the dynamic behaviours of a typical portable electronic device under drop impact loading. Firstly, an idealized system which contains an outer case and a PCB attached with a package was adopted as specimen. With an innovative design, the actual impact force pulses were measured by employing a Hopkinson bar in the dynamic test rig. Dynamic strains of the PCB were simultaneously recorded to explore the correlation between the strains and the impact pulse. Particular attention has been paid to the dependence of the shock response of the PCB on the impact velocity, impact force pulse, as well as the impact orientation. Analysis is carried out to explain the experimental results. A deep understanding of the shock response of typical electronic product systems will help to guide the design of rugged and highly impact-resistant devices
\end{abstract}

Keywords: portable electronic products, drop test, impact.

\section{Introduction}

Benefit from the advancements of new technologies, electronic components have become smaller, thinner and lighter. Today, portable electronics, such as cellular phones, PDAs (personal digital assistants), digital cameras etc., have a 
substantial market share worldwide. It should be noted, however, that portable devices are often exposed to relatively harsh operating environments due to the likelihood of dropping these devices and also making impact with other objects. And drop/impact induces failure is one of the most dominant causes of damage for portable devices. Therefore, drop impact reliability is one of the major concerns in product design.

Traditionally, the rigidity of products is obtained through a design-failureredesign process. It is evident from engineering practice that in order to improve the shock protection of portable electronics, instead of making protective designs on a trial-and-error basis, one must understand how electronic components or portable electronic devices respond to shock and impact loading. And drop testers were firstly designed by researchers to assess drop/impact performance of the products. Jason Wu et al. [1] pointed out that drop tests through hand-drop and eye-check can only give a qualitative but no quantitative information about impact behavior. They introduced a drop tester which can control drop orientation by two slide cables. In order to control the orientation of the object at impact and to instrument the drop tests, S. Goyal and E. K. Buratynski [2] also proposed a method that the specimen be suspended onto a guided drop-table in desired orientation. The drop-table hits the ground first, but just before impact, the test object is released from suspension so it can move unrestrained thereafter. V.P.W. Shim and C. T. Lim [3] patented an impact drop tester which consisted of a pair of grippers mounted on a sliding block. The grippers can grip the specimen in any specified orientation. This block is then hoisted to the desired drop height and released to undergo free fall. Just prior to impact, the product is quickly released from the grippers at the specified orientation.

Comprehensive tests were conducted by Seah et al. [4] and Lim C. T. et al. $[5,6]$ to investigate drop impact response of several models of PDAs and mobile phones. Impact force, strains and level of acceleration induced at the PCB were measured and compared in different orientations.

However, most of previous experiments were aimed to study some specified model of products. Although their results offered the original information about the strains and impact forces during drop impact, up to now, none systematic measurements were carried out to answer some fundamental problems, for example, how the dynamic responses of the devices (say maximum strain and impact force) changes with impact parameters (say drop orientation and impact velocity) and how the impact wave propagates from the outer case to inner structures. Another point is that due to the wave propagations and reflects, most of commercial load cells for drop impact may not give precise force impulse of the impact. This paper aims to record in detail the process of the impact wave propagation and discuss in a general way the effect of impact parameters on dynamical response of a typical electronic device.

Firstly, an idealized system which contains an outer case and a PCB attached with a package was adopted as specimen. With an innovative design, the actual impact force pulses were measured by employing a Hopkinson bar in the dynamic test rig. Dynamic strains of the PCB were simultaneously recorded to explore the correlation between the strains and the impact pulse. Particular 
attention has been paid to the dependence of the shock response of the PCB on the impact velocity, impact force pulse, as well as the impact orientation. Analysis is carried out to explain the experiments results. A deep understanding of shock response of typical electronic product systems will help to guide the design of rigged and highly impact-resistant devices.

\section{Experimental set-up}

The drop test system, see figure 1, consists of a pendulum system and a PVC bar. The PVC bar is adopted to record the impact pulse. A 180 degrees air gripper MHY2, produced by SMC Corporation, is assembled at the end of a $1.5 \mathrm{~m}$ pendulum arm. This air gripper can be rotated and then fasted to hold the specimen in any desired orientation. During tests, the gripper together with specimen is swung to a specified height then set free. A pair of laser beam and laser detector together with an electronic control system is designed to open the gripper and release the specimen just before it hit the end of the horizontal bar. Therefore, the specimen will impact with the horizontal bar in desired orientation and the gripper will swing away freely. Red ink was marked on the bar end to record the contact point of every test.

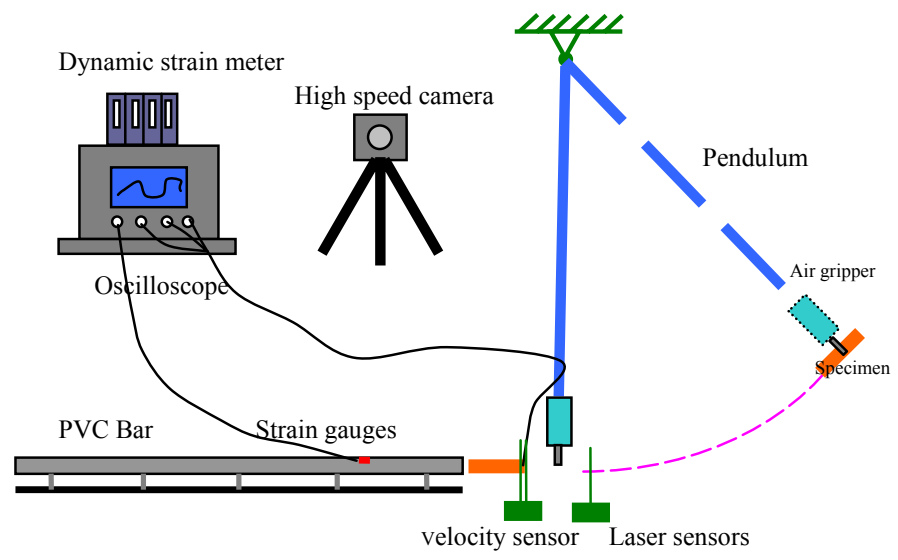

Figure 1: $\quad$ Schematic illustration of the drop tester.

The PVC bar has a diameter of $26 \mathrm{~mm}$. Since the ordinary impact pulse duration is less than $1.2 \mathrm{~ms}$ and the measured wave speed is $1730 \mathrm{~m} / \mathrm{s}$, to guarantee that the impact pulse should not be interfered by reflection from the far end, the length of the bar is chose to be $2 \mathrm{~m}$. In order to get rid of bending effect on the bar, four strain gauges are attached around a circle to record the compression wave.

To simplify the specimen structure, the typical electronic device designed, shown in figure 2, is composed by a PBC and an outer case. The 
$110 \mathrm{~mm} \times 60 \mathrm{~mm} \times 20 \mathrm{~mm}$ outer case is made of PVC. The thickness is $5 \mathrm{~mm}$. A $40 \mathrm{~mm} \times 90 \mathrm{~mm}$ single layer PCB is fasted on the case by four screws at four corners. A 64 balls $8 \mathrm{~mm} \times 8 \mathrm{~mm}$ BGA package with pad size $0.4 \mathrm{~mm}$ pitch $0.8 \mathrm{~mm}$ is attached to the center of the PCB.
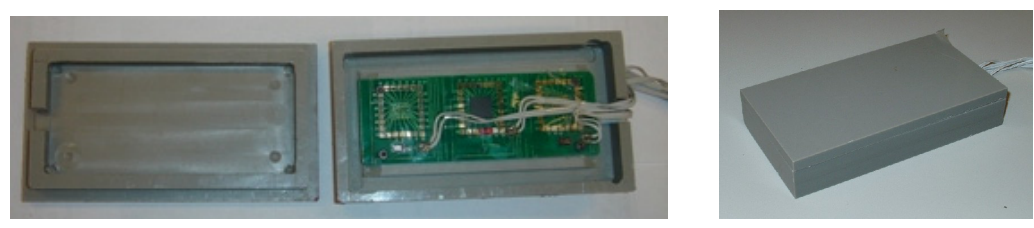

Figure 2: Photographs of the specimen.

Four strain gauges were used to measure the longitudinal strains on the $\mathrm{PCB}$, see figure 3. Since it is reported that the failure of the solder joint is mainly because the bending of the PCB. One stain gauge was attached near the chip and another one is attached to the opposite face at the same point. And the other two strain gauges were attached near the screw to test the wave transferred from the outer case. At the same time, in order to measure the impact wave on the outer case, two more strain gauges were attached in longitudinal direction on the case.
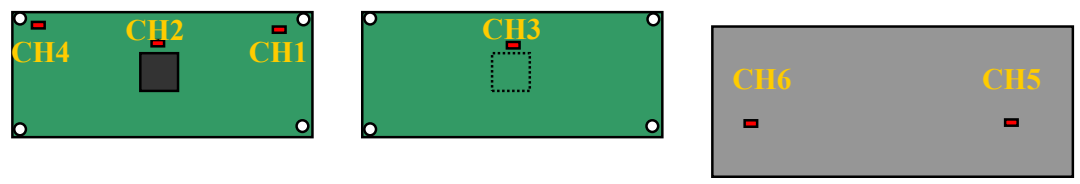

Figure 3: Schematic diagram showing strain gauge locations.

\section{Repeatability and reliability of the system}

Though random drops of the specimen by hand, it was found that there was hardly any chance that the specimen may contact with the ground with one of its lines or one of its faces. Therefore, in our experiments the specimen hitting the bar end is capable of mimicking the point contact in real drop. The photographs taken by the high speed camera see figure 4 , proved that there is not any change on the orientation after the specimen is released by the air gripper.

To ensure the test consistence, three to five drops were performed for every specified situation. The results demonstrate that both impact force measurements and strain measurements are highly repeatable, as shown in Figure 5. From the signal obtained from the bar, it is evident that the input impact wave and the reflection of the wave were successfully separated. The reflective tension wave obtains the measure point after $2 \mathrm{~ms}$ while the impact was finished less than $1.2 \mathrm{~ms}$. 

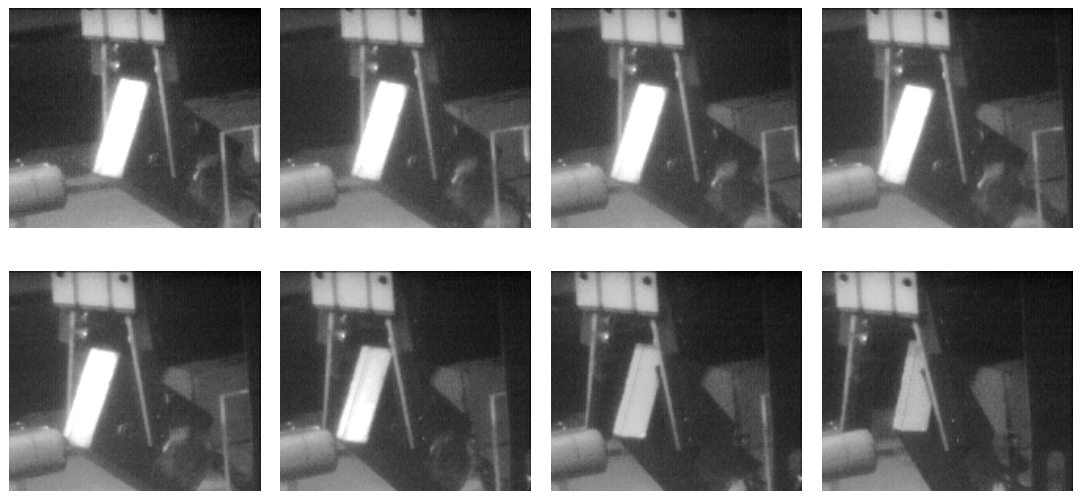

Figure 4: $\quad$ Impact process taken by high speed camera.
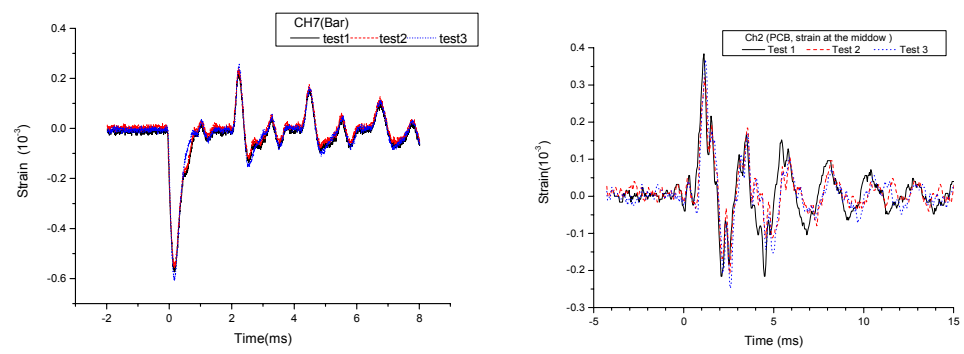

Figure 5: $\quad$ Repeatability of impact pulse and strain on PCB.

\section{Experimental results}

\subsection{Impacts in different orientations}

Denote the length, width and thickness of the specimen with $a, b$ and $c$ separately. We choose the angle $\alpha$ and $\gamma$ as the two angles which are necessary for determination of the impact orientation. Where $\alpha$ is the angle between the projection of the bar axis in the main face $(a \times b)$ and the line $a$, and $\gamma$ is the angle between the bar axis and the $c$ direction. The orientations in which the drop tests are conducted are illustrated in figure 6 below.

Because the thickness of the device is much shorter than its length and width, we chose $\gamma$ as the major variable parameters when considering the orientation effect. And it is clear from classical mechanics that when the axis of the bar passes through the central of the devices, the impact impulse should be higher than other conditions. Therefore, we fixed the angle $\alpha$ at $26^{\circ}$, that is the angle of the diagonal of the main face to line $a$. And for every test, the drop velocity is $3.53 \mathrm{~m} / \mathrm{s}$. 


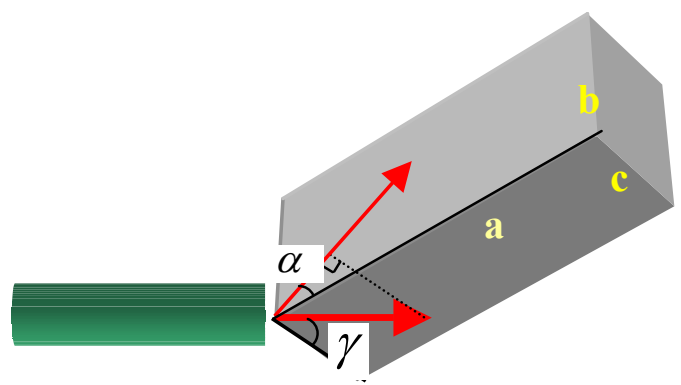

Figure 6: Orientation parameters.

Figure 7a-g illustrates the dynamic strain response with different angle $\gamma$. Figure 8 plots the peak value of strain with respect to angle $\gamma$. It is interesting that from the test results: (1)Ch1 has the highest strain on PCB, however point Ch1 is the most far from the hitting point; (2) with the change of angle, $\gamma$, the phase and peak value of the strain on PCB and strain on the outer case have no significant variation. (3) The impact force increase with $\gamma$

The spectrum analysis, given by Table 1 indicates that the basic frequencies of the outer case are $1099 \mathrm{~Hz}$ and $1587 \mathrm{~Hz}$, while the basic frequencies of the $\mathrm{PCB}$ are $488 \mathrm{~Hz}$ and $854 \mathrm{~Hz}$. For $\mathrm{Ch} 1$ and $\mathrm{Ch} 4$ where the strain gauges near the screw points, the response contains both the basic frequencies of $\mathrm{PCB}$ and that of the outer case. For $\mathrm{Ch} 2$ and $\mathrm{Ch} 3$, the response can be substitute into two parts: flexural wave with frequencies $488 \mathrm{~Hz}$ and $854 \mathrm{~Hz}$ represented by $\mathrm{Ch} 2-\mathrm{Ch} 3$, and a small part of compression wave of $1656 \mathrm{~Hz}$ represented by $\mathrm{Ch} 2+\mathrm{Ch} 3$. According to the spectrum analysis, wave propagation of the outer case has very low effect to the dynamic performance of the central part of PCB.

\subsection{Impacts under different velocities}

For the following group of test, we are aimed to investigate the effect of drop height or drop velocity to the dynamic response of the device. We constrained the impact orientation at $\alpha=26^{\circ}$ and $\gamma=60^{\circ}$, then gradually increase the drop height from $0 \mathrm{~m}$ to $1.5 \mathrm{~m}$. Major concern is the peak values of the strains, which is reported essential for the failure of the solder ball.

Figure 9 to 10 plots the peak strains on PCB, peak strain on the outer case the peak impact load with respect to velocity change.

An interesting phenomenon is illustrated by figure 9a that the peak strains on PCB may stay on a stable plateau after the impact velocity reached a certain level. This information is highly valuable. Once we understand which factors affect the level of plateau, we can propose effective methods to protect the PCB. 


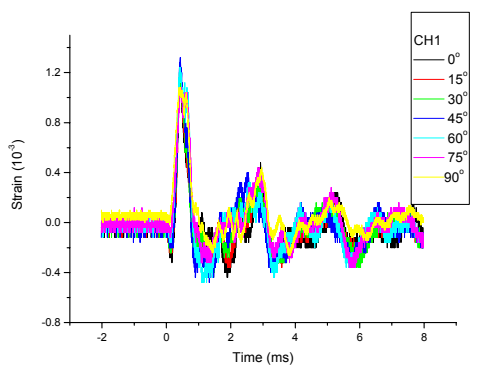

a

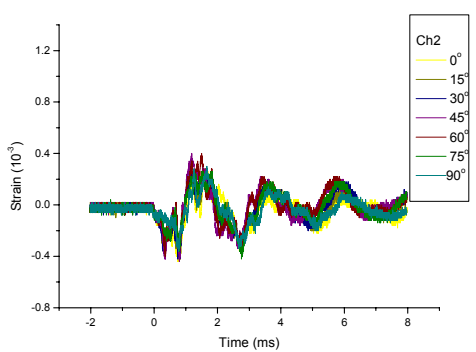

$\mathrm{c}$

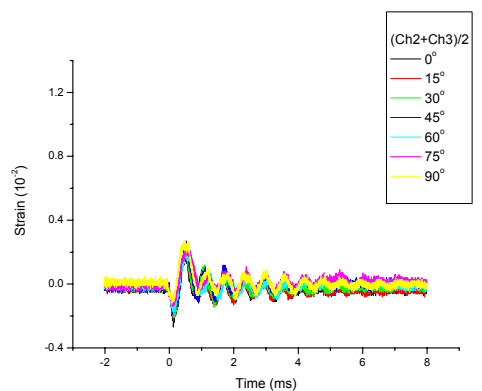

e

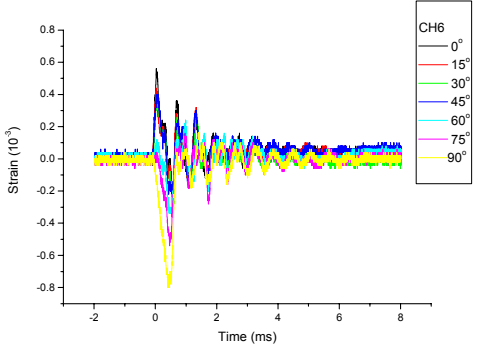

g

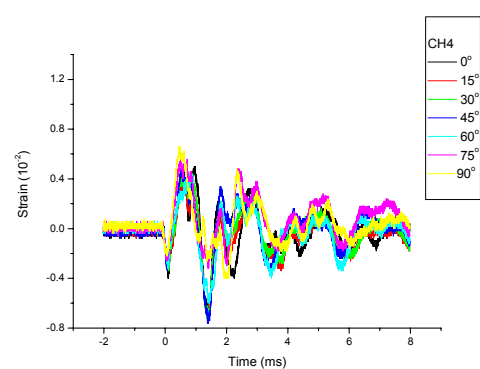

b

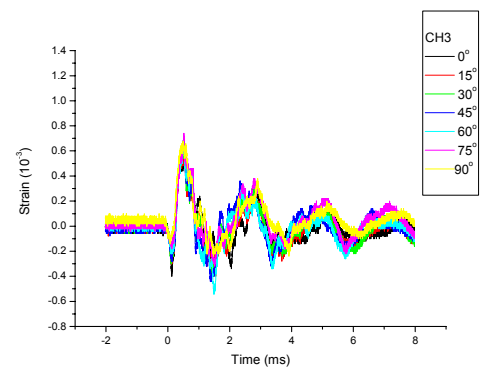

d

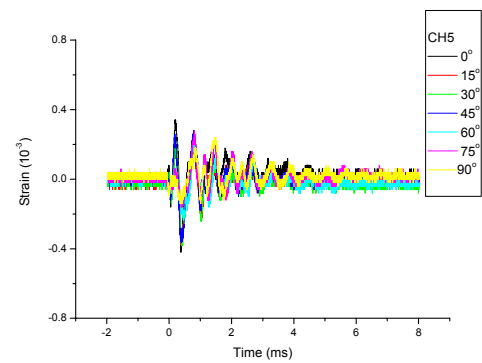

f

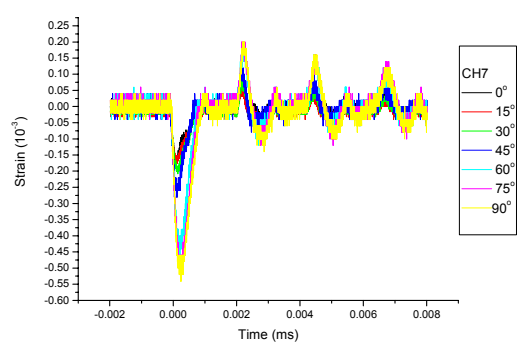

$\mathrm{h}$

Figure 7: Impact response with respect to time. 


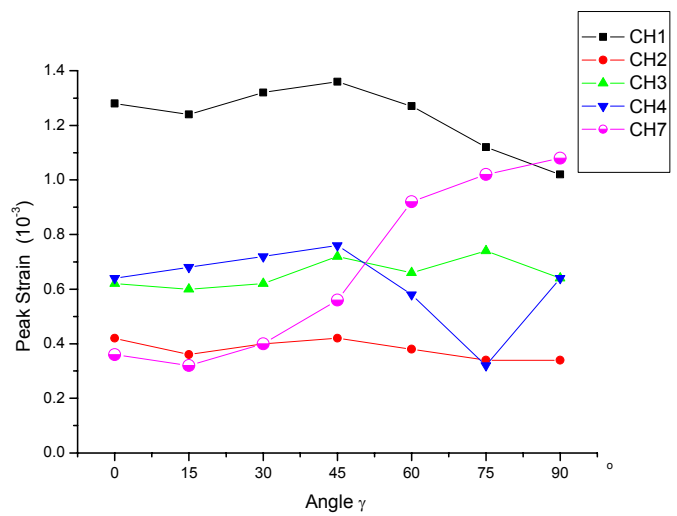

Figure 8: $\quad$ Peak Strains with respect to angle $\gamma$.

Table 1: $\quad$ Spectrum of impact responses.

\begin{tabular}{|c|c|c|c|c|}
\hline & 1 & 2 & 3 & 4 \\
\hline Ch1 & $488 \mathrm{~Hz}$ & $854 \mathrm{~Hz}$ & $1099 \mathrm{~Hz}$ & $1587 \mathrm{~Hz}$ \\
\hline Ch4 & $488 \mathrm{~Hz}$ & $854 \mathrm{~Hz}$ & $1587 \mathrm{~Hz}$ & -- \\
\hline Ch2+Ch3 & $1656 \mathrm{~Hz}$ & -- & -- & -- \\
\hline Ch2-Ch3 & $488 \mathrm{~Hz}$ & $854 \mathrm{~Hz}$ & -- & -- \\
\hline Ch5 & $1099 \mathrm{~Hz}$ & $1587 \mathrm{~Hz}$ & $3296 \mathrm{~Hz}$ & -- \\
\hline Ch6 & $1587 \mathrm{~Hz}$ & $3296 \mathrm{~Hz}$ & -- & -- \\
\hline
\end{tabular}

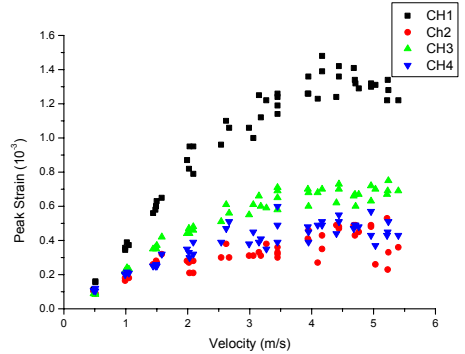

(a)

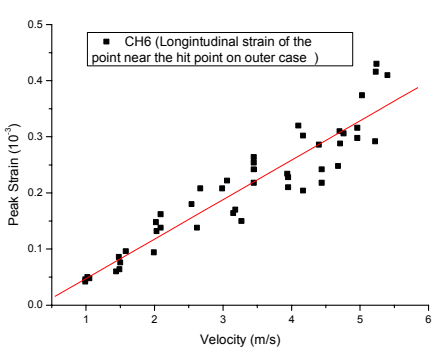

(b)

Figure 9: (a)Peak Strains on PCB with respect to impact velocity (b) Peak Strains on the outer case with respect to impact velocity.

According to figure $9 \mathrm{~b}$, the peak strain of the outer case responses linearly to the impact velocity. From the results plotted by figure 10, it can also be concluded that the peak load is proportional to the impact velocity. This means the momentum of impact (correlated to impact velocity) rather than the impact energy (correlated to drop height) is the major control parameter for impact force. 


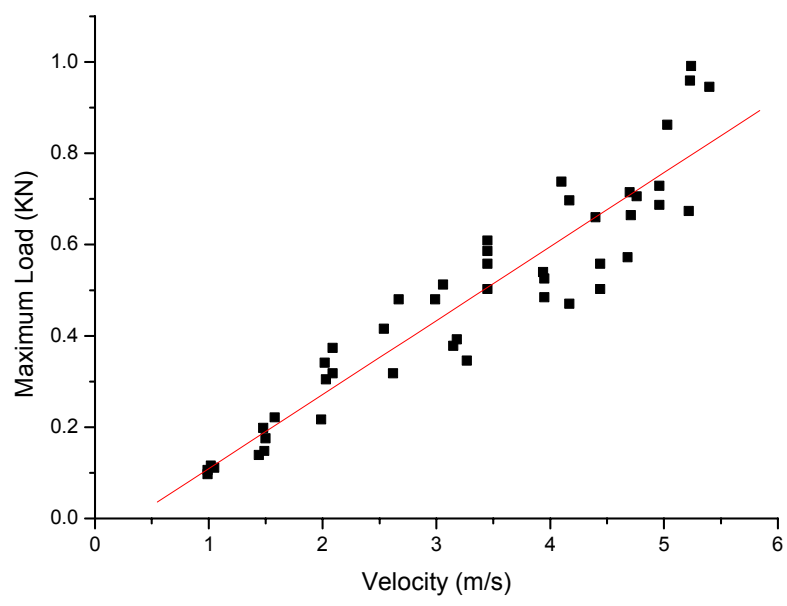

Figure 10: Peak impact load with respect to impact velocity.

\section{Conclusions}

A drop test system was designed to perform repeatable drop tests with any specified orientation. With an innovative design, the actual impact force pulses were measured by employing a Hopkinson bar in the dynamic test rig.

Although the impact force increased a lot when impact angle $\gamma$ changes from 0 to 90 degrees, no significant changes were observed for responses on PCB and outer case.

Spectrum analysis of the signals shows that near the screw points, the response of PCB may interfere with outer case. However, at the central part, the dynamic responses were controlled by PCB itself. Both flexural waves and in plane compressive waves were detected near the chip.

Impact peak load is proportional to the impact velocity. The peak response of outer case is also linear to impact velocity.

When impact velocity reaches a certain value, the peak stain on the PCB may stay in a plateau stage and no longer increase linearly with the impact velocity.

\section{Acknowledgements}

This project is funded by Hong Kong RGC (Research Grants Council), Ref No. HKUST6189/03E. The authors like to express their appreciation to the support from HK RCG. 


\section{Reference}

[1] Wu, J.; Song, G.; Yeh, C.-P.; Wyatt, K.; Drop/impact simulation and test validation of telecommunication products, Thermal and Thermomechanical Phenomena in Electronic Systems. ITHERM '98. The Sixth Intersociety Conference on, 27-30 May 1998, pp330 -336, 1998.

[2] Goyal, S. and Buratynski, E.K., Methods for realistic drop-testing, Int. J. Microcircuits and Electronics Packaging (IJMEP), 23(1), pp45-52, 2000.

[3] Shim, V. P. W. and Lim, C.T., Impact Drop tester for Portable Consumer Products, US Patent No. 09/592,262 filed on 13 June 2000.

[4] Seah, S.K.W.; Lim, C.T.; Wong, E.H.; Tan, V.B.C.; Shim, V.P.W.; Mechanical response of PCBs in portable electronic products during drop impact, Electronics Packaging Technology Conference. 4th, 10-12 Dec. 2002, pp $120-125,2002$.

[5] Lim, C.T. and Low, Y.J., Investigating the drop impact of portable electronic products. New Jersey: IEEE, Inc, 52 Electronic Components \& Technology Conference, United States, pp1270-1274, 2002.

[6] Lim, C.T.; Ang, C.W.; Tan, L.B.; Seah, S.K.W.; Wong, E.H.; Drop impact survey of portable electronic products, Electronic Components and Technology Conference, 2003. Proceedings 53rd, May 27-30, pp 113 120, 2003. 